\title{
A study on the use of smartphones under realistic settings to estimate road roughness condition
}

\author{
Viengnam Douangphachanh ${ }^{*}$ and Hiroyuki Oneyama
}

\begin{abstract}
Almost every today's smartphone is integrated with many useful sensors. The sensors are originally designed to make the smartphones' user interface and applications more convenient and appealing. These sensors, moreover, are potentially useful for many other applications in different fields. Using smartphone sensors to estimate road roughness condition has a great potential, since many similar sensors are already in use in many sophisticated road roughness profilers. This study explores the use of data, collected by sensors from smartphones under realistic settings, in which the smartphones are placed at more realistic locations and under realistic manner inside a moving vehicle, to evaluate its relationship with the actual road pavement roughness. An experiment has been conducted to collect data from smartphone acceleration and Global Positioning System (GPS) sensors; frequency domain analysis is also carried out. It has been revealed that the data from smartphone accelerometers has a linear relationship with road roughness condition, whereas the strength of the relationship varies at different frequency ranges. The results of this paper also confirm that smartphone sensors have a great potential to be used for estimating the current status of the road pavement condition.
\end{abstract}

Keywords: Smartphone sensors; Road roughness; Roughness condition; Condition estimation; Realistic settings

\section{Introduction}

Maintaining and monitoring road infrastructure is a challenging task for almost all governments and road authorities. One of the reasons is that the task requires the collection of substantial amount of road network condition data, which is very important for the maintenance planning and monitoring, over time, in addition to the significant efforts that have to be directed to actual maintenance of the road network. In developing countries, the attention that should be addressed on data collection is usually ignored or neglected mainly due to the lack of technology and budget. Therefore, in these countries, road infrastructure condition data is often left outdated, making it difficult for proper planning and programming of the maintenance.

'Road Roughness is consistently recognized as one of the most important road condition measures throughout the world. The time series recording of roughness data allows pavement managers to assess the roughness progression rate of pavements and to take appropriate action

\footnotetext{
* Correspondence: douangphachanh-viengnam@ed.tmu.ac.jp Department of Civil and Environmental Engineering, Tokyo Metropolitan University, 1-1 Minami-Osawa, Hachioji-shi, Tokyo 192-0397, Japan
}

accordingly' [1]. International Roughness Index (IRI) is an indicator that is widely adopted to classify road roughness condition, which has been used widely for road infrastructure maintenance and monitoring for many decades [2]. IRI is the condition index obtained from the measurement of longitudinal road profiles with the measuring unit of slope $(\mathrm{mm} / \mathrm{m}, \mathrm{m} / \mathrm{km}$ for instance). To measure IRI, there are many approaches; however, majority of them, on the one hand, requires sophisticated profilers and tools, which are expensive to acquire and operate as well as often require skillful operators. On the other hand, visual inspection is also a popular practice in many developing countries. While this is relatively a much cheaper option to implement, it is usually very labor intensive and time consuming.

Using smartphones to collect the data is a promising alternative because of its low cost and easy to use feature in addition to its potentially wide population coverage as probe devices. In our previous study [3], we explored the use of smartphones, fixed to vehicles with predetermined orientation, to estimate road roughness where promising results have been observed. In order to find our new features and compare the accuracy of the estimation,

\section{穴}






Figure 1 VIMS components.

this study will take a further step by attempting to estimate road roughness condition from smartphones under more realistic settings, which is beyond fixed orientation and/or fastening the devices themselves tightly with vehicles while collecting data. In other words, the smartphone are placed loosely at locations that a driver would be more likely to put their smartphones inside a car while driving.

\section{Related work}

There are very few studies that have directly explored the use of smartphone to estimate IRI of road pavement. In previous studies, while there is a lot of interest in detecting road bumps and anomalies using mobile sensors, majority of them focus on identifying and locating road bump and anomalies instead of estimating road pavement condition, particularly in terms of IRI measurement and/ or estimation. The most relevant work to our study includes the use of a stand-alone accelerometer to fit in a simulation car and use it to assess road roughness condition [4]. The simulations in this study conclude that roughness of the road can be estimated from acceleration data obtained from the sensor. Similarly, in another study [5], a system has been developed to utilize stand-alone accelerometers to successfully detect road anomalies. In India, a group of researchers use many sensing components from a mobile phone such as accelerometer, microphone, Global System for Mobile communications (GSM) radio, and Global Positioning System (GPS) to monitor road and traffic conditions [6]. By analyzing data from the sensors, potholes, bumps, braking,

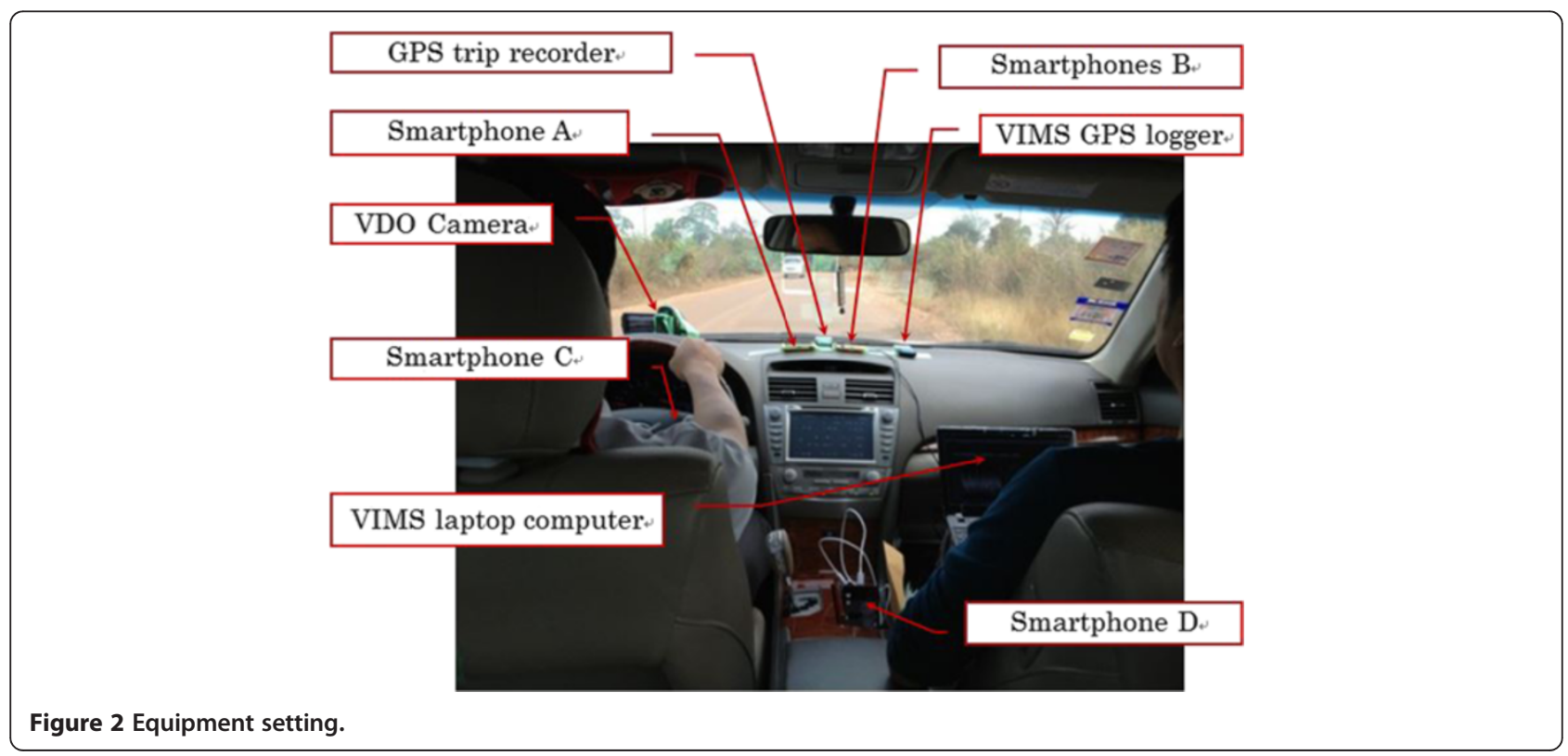


Table 1 Experiment arrangement

\begin{tabular}{|c|c|c|}
\hline Experiment date & $\begin{array}{l}\text { Vehicle and } \\
\text { equipment }\end{array}$ & $\begin{array}{l}\text { Location of } \\
\text { smartphone }\end{array}$ \\
\hline \multirow[t]{3}{*}{17 to 18 Nov 2012} & Vehicle 1 & \\
\hline & Smartphone C & Pocket \\
\hline & Smartphone D & Near gearshift \\
\hline \multirow[t]{3}{*}{19 to 20 Nov 2012} & Vehicle 2 & \\
\hline & Smartphone C & Pocket \\
\hline & Smartphone D & Near gearshift \\
\hline \multirow[t]{3}{*}{21 Nov 2012} & Vehicle 1 & \\
\hline & Smartphone C & Near gearshift \\
\hline & Smartphone D & Pocket \\
\hline \multirow[t]{3}{*}{22 Nov 2012} & Vehicle 3 & \\
\hline & Smartphone C & Pocket \\
\hline & Smartphone D & Near gearshift \\
\hline \multirow[t]{3}{*}{23 Nov 2012} & Vehicle 4 & \\
\hline & Smartphone C & Pocket \\
\hline & Smartphone D & Near gearshift \\
\hline
\end{tabular}

and honking can be detected. The information is then used to assess road and traffic conditions. In [7] and [8], Android smartphone devices with accelerometers are used to detect location of potholes. Their approach includes many simple algorithms to detect events in the acceleration vibration data. In [9] and [10], the authors analyze data obtained by smartphone accelerometers in frequency domain to extract features that are corresponding to road bumps. In Japan, a group of researchers has developed an Android smartphone application called 'BumpRecorder' [11] to detect the location and severity of road bumps on road networks that have been affected by the March 11, 2013, earthquake in Tohoku region, Japan.

\section{Approach}

\subsection{Experiment}

An experiment has been conducted in Vientiane, Laos, in November 2012, to collect data for our analysis. Our initial assumption is that the vibration of vehicles may be different at different road sections depending on roughness conditions of the pavement, and by placing smartphones with relevant sensors in the vehicles, the vibration signal could be captured. With the assumption, we place smartphones and other equipment inside experiment vehicles and drive along selected roads to collect data for our analysis.

Main equipment used in this experiment includes two smartphones, a Samsung Galaxy Note 3 (GT-N7100; Samsung Electronics Co., Ltd., Suwon, Korea) and an LG 4X HD (LG-P880; LG Electronics, Seoul, Korea), a GPS trip recorder (747Pro; Transystem Inc., Hsinchu, Taiwan), and a Sony video camera (Sony Corporation, Minato, Japan).

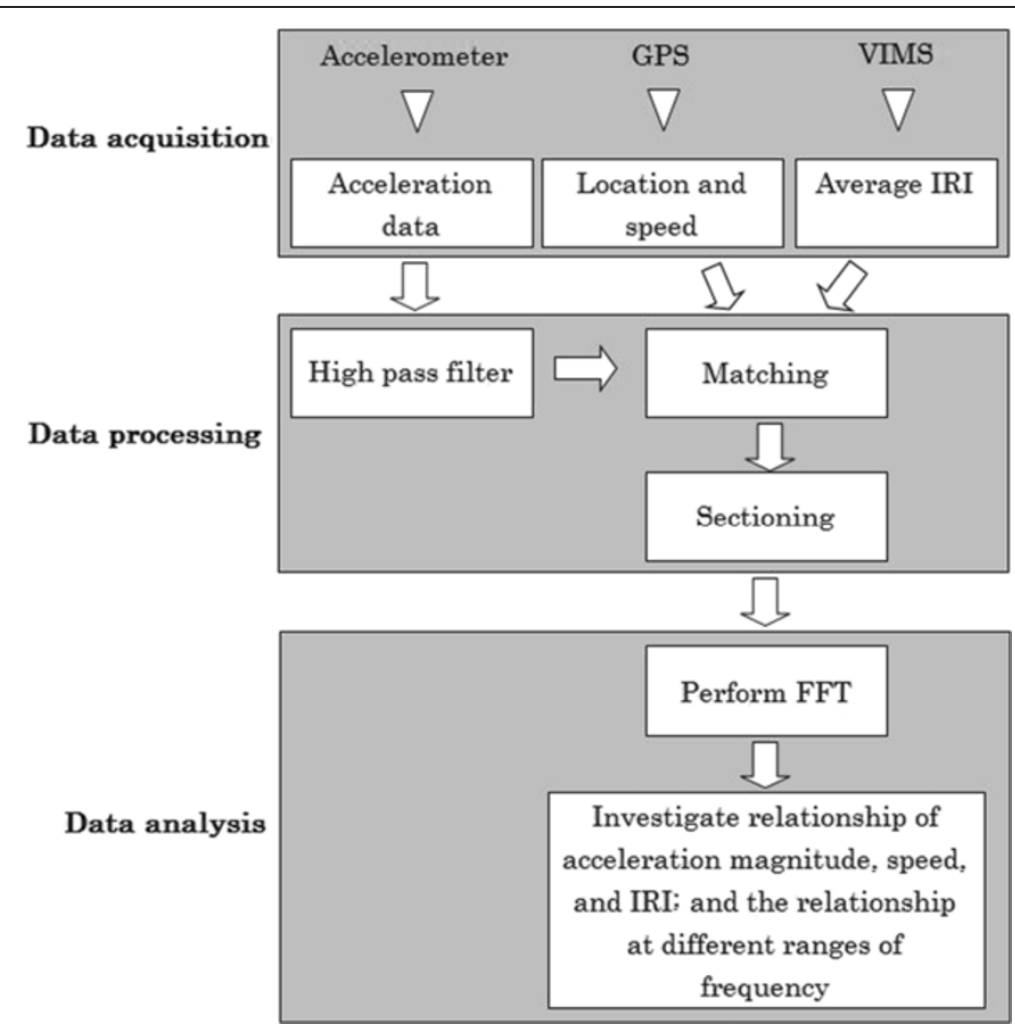

Figure 3 Data processing and analysis flowchart. 
The smartphones are pre-installed with an application called AndroSensor [12]. The application is used to record only acceleration data $(\mathrm{x}, \mathrm{y}, \mathrm{z})$ from accelerometer, and location data (including speed) from GPS is needed. Data recording is done at an interval of $0.01 \mathrm{~s}$ or at a frequency rate of $100 \mathrm{~Hz}$.

The road routes selected for the experiment include various sections with different pavement roughness conditions ranging from good $(0 \leq \mathrm{IRI}<4)$, fair $(4 \leq \mathrm{IRI}<7)$, poor $(7 \leq \mathrm{IRI}<10)$, and bad (IRI $\geq 10)$. These condition classifications are based on condition indices used in the Lao Road Management System.

Referenced pavement condition data for this study is also obtained using Vehicular Intelligent Monitoring System (VIMS) [13]. VIMS comprises of both hardware and software. The hardware includes a laptop computer, a data acquisition module, an accelerometer, and a GPS logger (Figure 1). All the components are connected to each other via cables. The VIMS software includes two main programs: an application for calibration and data collection and an application to carry out the analysis. The system calculates the International Roughness Index (IRI) for every $10-\mathrm{m}$ road section. The main limitation of VIMS is that it cannot estimate IRI of road sections where the travel speed of the experiment vehicle is less than $20 \mathrm{~km} / \mathrm{h}$.

In our experiment setting, we use the smartphones to place at two different locations, one inside the driver's shirt front pocket and the other one in a box near the gearshift (Figure 2), inside an experiment vehicle. Unlike in our previous experiment, where we also used two smartphones to glue tightly on the experiment vehicles' dashboard (assuming that the orientation of the smartphones is fixed; Smartphone A and Smartphone B in

Table 2 Road sections selected for the analysis

\begin{tabular}{lcc}
\hline & $\begin{array}{c}\text { Number of sections } \\
\text { selected for analysis }\end{array}$ & $\begin{array}{c}\text { Location of } \\
\text { smartphone }\end{array}$ \\
\hline Vehicle 1 & & \\
\hline Smartphone C & 311 & Pocket \\
\hline Smartphone D & 492 & Near gearshift \\
\hline Smartphone C & 246 & Near gearshift \\
\hline Smartphone D & 320 & Pocket \\
\hline Vehicle 2 & & Pocket \\
\hline Smartphone C & 467 & Near gearshift \\
\hline Smartphone D & 592 & Pocket \\
\hline Vehicle 3 & & Near gearshift \\
\hline Smartphone C & 309 & \\
\hline Smartphone D & 421 & Pocket \\
\hline Vehicle 4 & & Near gearshift \\
\hline Smartphone C & 382 &
\end{tabular}

Figure 2), the two smartphones in this experiment are allowed to move freely inside the pocket and the box. The driver's shirt front pocket is not very likely to allow the smartphone to turn and thus change its orientation, although the smartphone can move accordingly with the driver's movement. The smartphone being placed inside the box near the gearshift, however, would be allowed to make a big change to its orientation due to the turn and vibration of the vehicle, although the change may not be a complete switch of the orientation from one axis to another.

Additionally, other equipment such as the GPS and the video camera are placed on the dashboard. VIMS
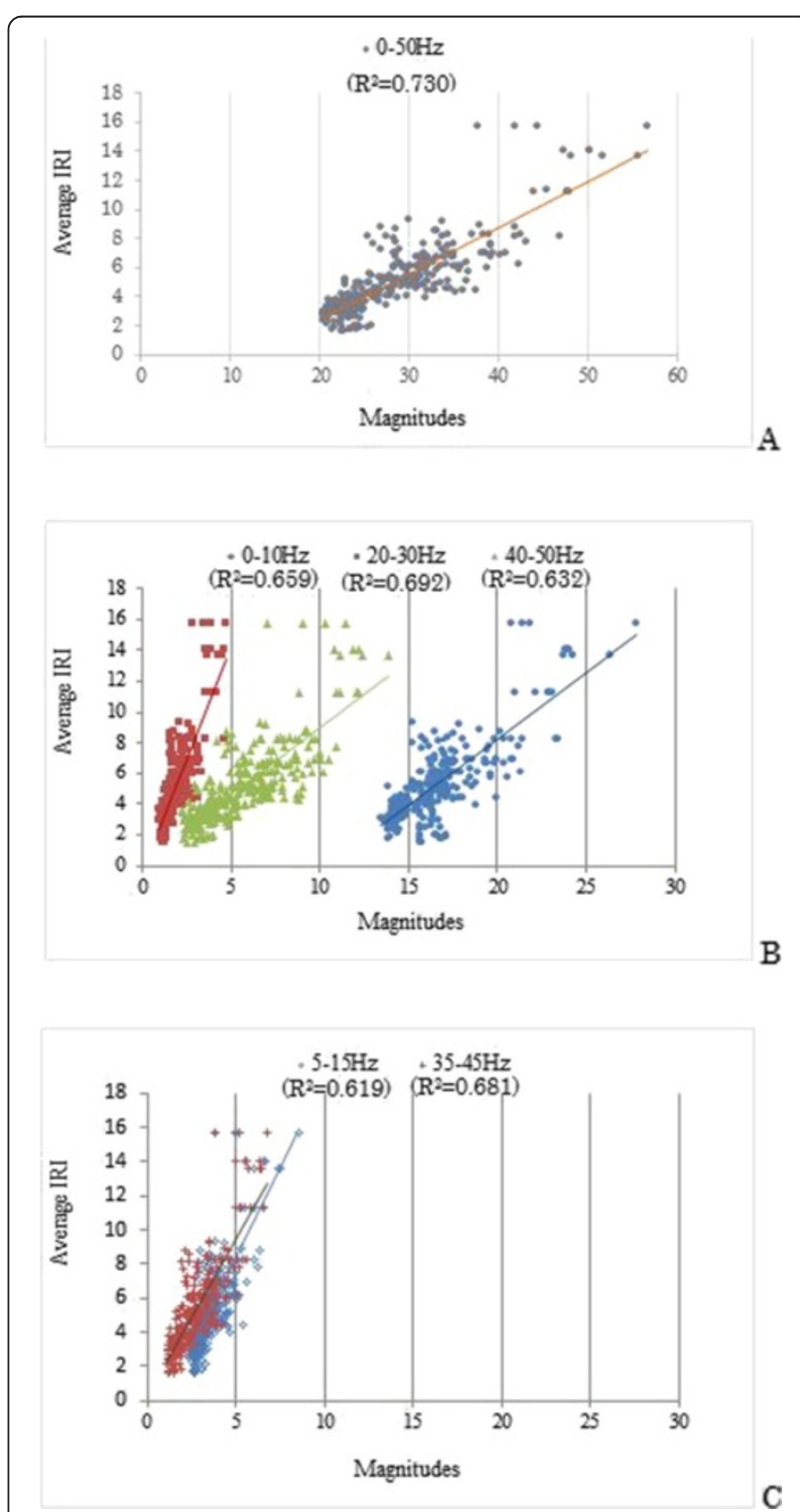

Figure 4 Selected results (A to C) from the previous study (vehicle 1, smartphone A). 


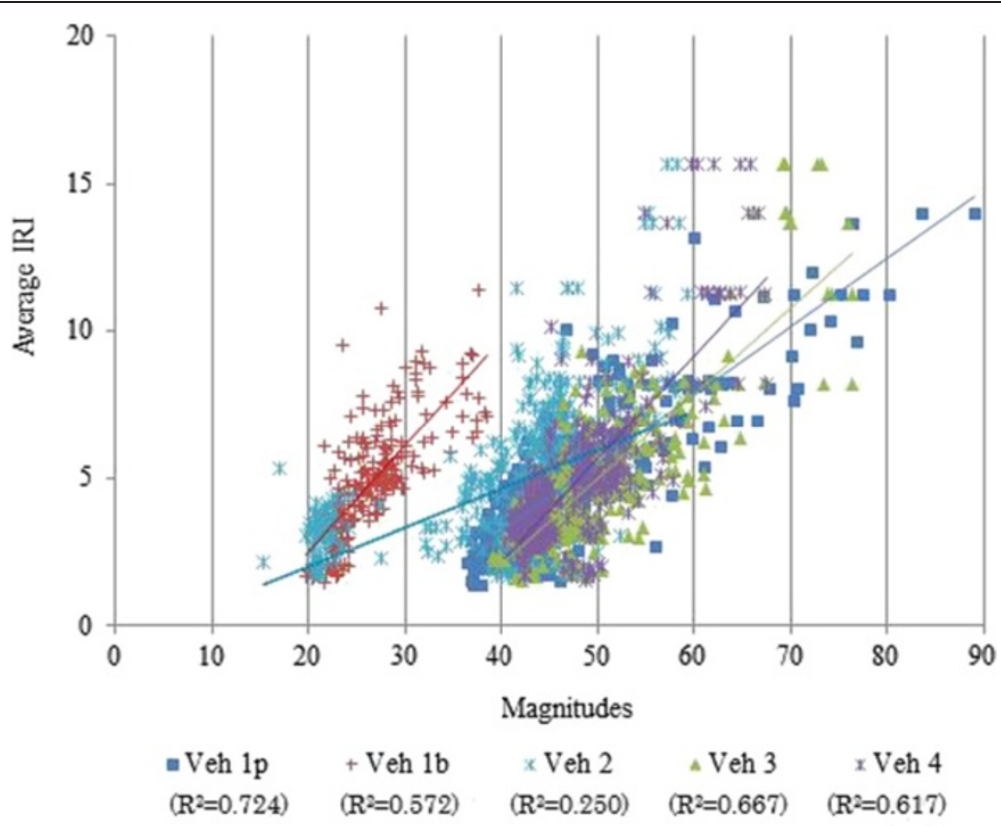

Figure 5 Road roughness condition (Average IRI) and acceleration data (Magnitudes) relationship for smartphone C in the total range of frequency $(0$ to $50 \mathrm{~Hz})$.

components are also installed in accordance to the VIMS manual [14].

The experiment arrangement can be summarized briefly in Table 1.

A total of four different vehicles are used for this experiment. Vehicle 1 is a Toyota Vigo 4WD pick-up truck (Toyota Motor Corporation, Toyota, Japan), vehicle 2 is a Toyota Camry sedan, vehicle 3 is a Toyota Vigo 2WD pick-up truck, and vehicle 4 is a Toyota Yaris sedan.

Note that in Figure 2, smartphone A and smartphone $B$ are only to show the smartphone setting in our previous experiment. The smartphone setting considered under this study is therefore only smartphone $\mathrm{C}$ and smartphone D.



Figure 6 Road roughness condition (Average IRI) and acceleration data (Magnitudes) relationship for smartphone D in the total range of frequency ( 0 to $50 \mathrm{~Hz}$ ). 
Smartphone $C$ is a Samsung Galaxy Note 3, and smartphone D is an LG 4X HD. Vehicle 1 is chosen for data collection on two separated runs, the first run on the 17 th to 18th and the second run on the 21st November 2012. On these two runs, the locations of smartphones $C$ and D are switched.

\subsection{Data processing and analysis}

In brief, data processing involves checking and filtering of the data collected by the smartphones and matching with referenced data before dividing into 100 -m sections (Figure 3). To remove unrelated low frequency signal, which is contributed by the effect of vehicle maneuver such as changing speed due to braking and turning as well as the contribution of the force of gravity, from all axes $(x, y$, and $z)$ of the acceleration data, we apply a simple high pass filter as recommended in the official Android Developer Reference [15]. A 100-m length of acceleration data is chosen as a unit for road roughness estimation in this study because (i) Road Management System in Laos requires road pavement condition to be estimated for every $100-\mathrm{m}$ section as it is believed to be convenient for maintenance planning, and (ii) there is also a concern on the accuracy of GPS position data; therefore, choosing a shorter section unit may cause some issues in data matching between VIMS and smartphone GPS data.

After sectioning, road sections that have incomplete data will be excluded from the analysis. The sections with incomplete data are those that have no data from VIMS, at the time when the experiment vehicle is travelling at a speed slower than that required by VIMS (less than $20 \mathrm{~km} / \mathrm{h}$ ) in traffic jam condition, for instance; and sections that have no GPS data, as sometimes GPS would fail to record information due to some satellite signal obstruction. Road sections where experiment vehicles have stopped (checking from speed and VIMS data) are also excluded since data at these sections cannot be used to estimate road roughness condition. In addition, sections that have the lengths that are $10 \%$ less or more than $100 \mathrm{~m}$, less than $90 \mathrm{~m}$, or more than $110 \mathrm{~m}$ are also omitted from the analysis.

Analysis is carried out in the frequency domain. Fast Fourier transform (FFT) is performed to calculate magnitudes for every selected 100-m section (Table 2) in two different arrangements, from (1) the sum of all three axis acceleration data (sum of $\mathrm{x}, \mathrm{y}, \mathrm{z}$ acceleration data) and (2) each axis of acceleration data ( $x, y, z$, separately). By considering all acceleration vibration from all three axes of the sensor, we assume that the effect of the smartphone orientation could be ignored. In other words, the smartphone would still be useful to estimate the roughness of the road surface regardless of its orientation and/or location settings. The two different arrangements is a further attempt to understand characteristics of acceleration data from the smartphone sensors. After that, we investigate the relationship between magnitude, speed, and IRI. FFT also allows us to study the mentioned relationship at different ranges of frequency, to see whether the sum of magnitudes at a particular range of frequency is more useful in expressing the road roughness condition or not.

\section{Results and discussion}

4.1 Correlation between the sum of magnitudes and IRI In our previous study [3], with the smartphones being fixed to the dashboard of the experiment vehicles, we
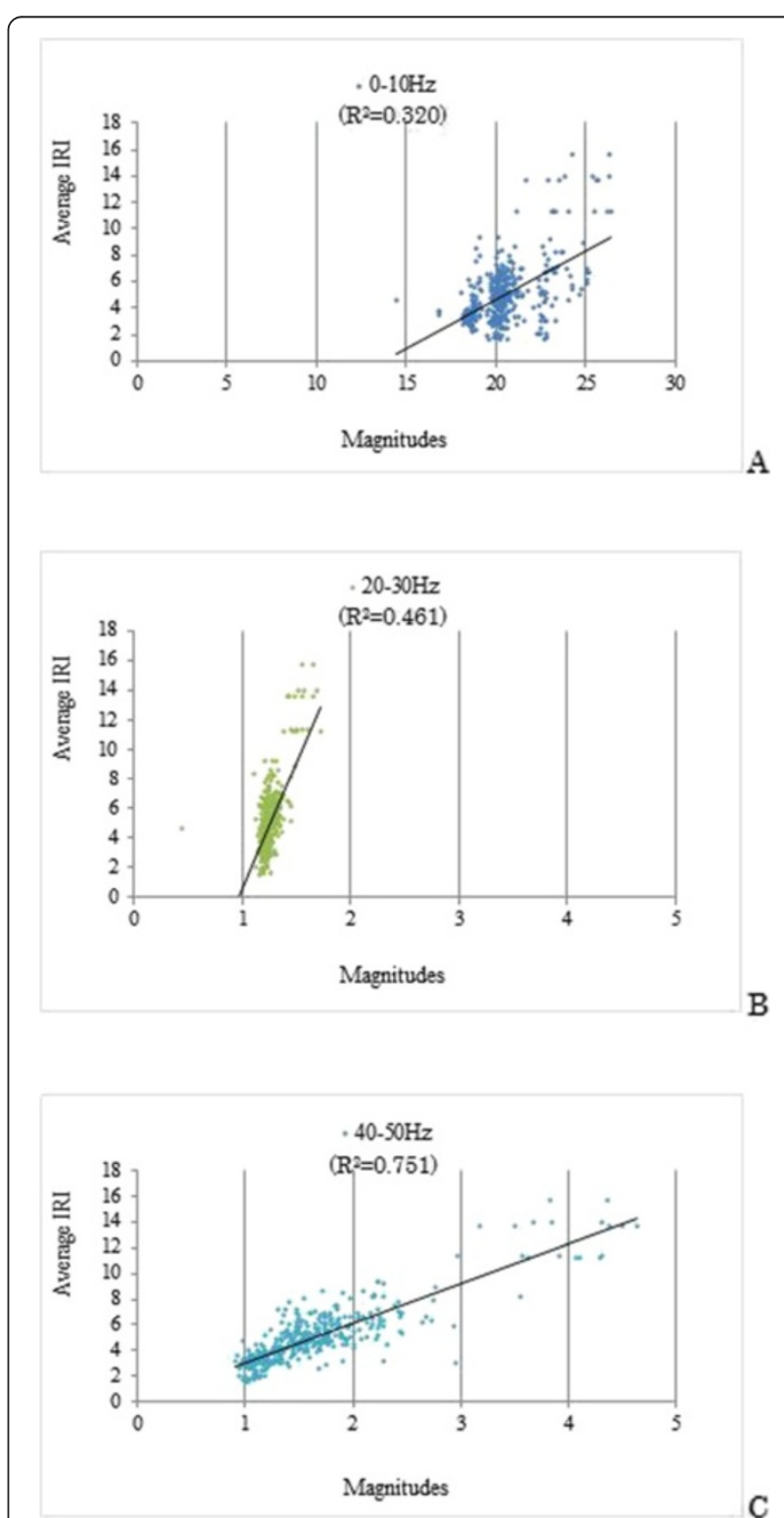

Figure 7 Relationship between road roughness condition (Average IRI) and acceleration data (Magnitudes) at different frequency ranges (A to C) (vehicle 3, smartphone $D$ ). 




Figure 8 Relationship of road roughness condition (Average IRI) and acceleration data (Magnitudes) for smartphone C in the frequency range 40 to $50 \mathrm{~Hz}$.

have observed that road roughness can be estimated from acceleration data. The relationship between the average IRI and the sum of magnitudes, calculated from the sum of all axes acceleration vibration, which is the only arrangement considered in [3], in the total range of frequency components, which is 0 to $50 \mathrm{~Hz}$ (half of the sample rate of $100 \mathrm{~Hz}$ ), is considerably significant, with the $R^{2}$ of as high as 0.730 and 0.647 for smartphones $\mathrm{A}$ and $\mathrm{B}$, respectively. The $R^{2}$ in the total range of frequency ( 0 to $50 \mathrm{~Hz}$ ) are generally slightly better than $R^{2}$ derived from the breakdown frequency ranges ( 0 to $10,10-20,20$ to 30,30 to 40 , and 40 to $50 \mathrm{~Hz}$; as well as 5 to 15,15 to 25,25 to 35 , and 35 to $45 \mathrm{~Hz}$ ) considered under the study. It is also interesting to note that all of the $R^{2}$ values, derived from the different breakdown frequency ranges, are considerably

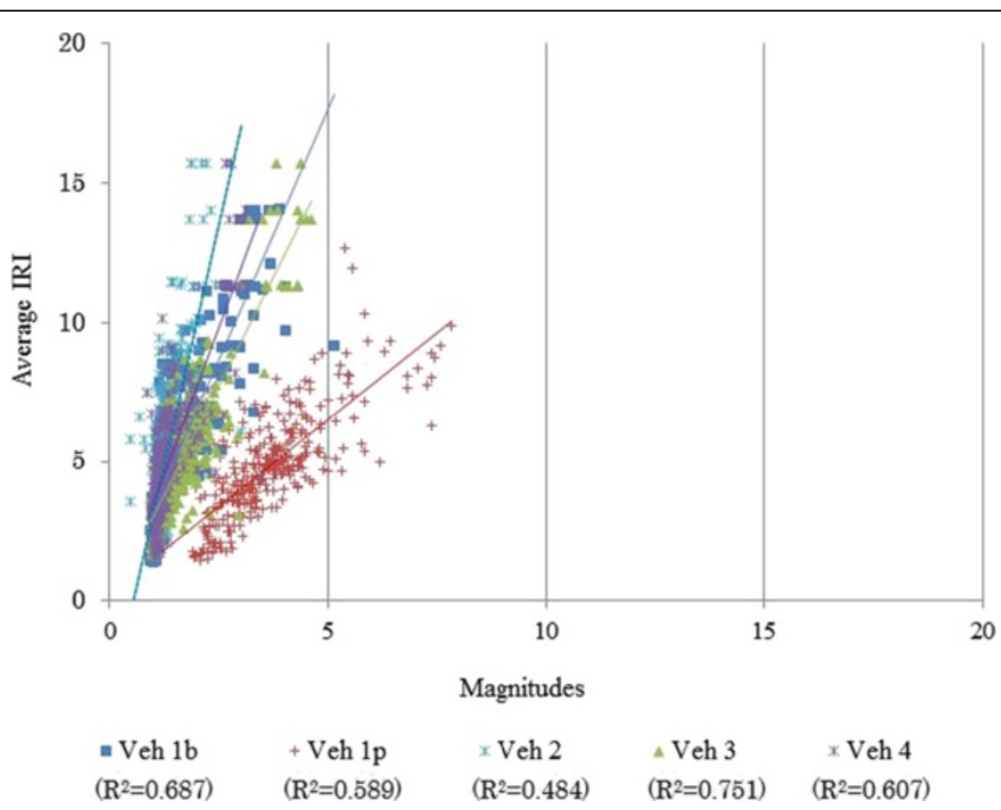

Figure 9 Relationship of road roughness condition (Average IRI) and acceleration data (Magnitudes) for smartphone D in the frequency range 40 to $50 \mathrm{~Hz}$. 
Table 3 A selected summary of the multiple regression analysis

\begin{tabular}{|c|c|c|c|c|c|c|c|c|}
\hline & \multicolumn{8}{|c|}{ Device D (box near gearshift) } \\
\hline & \multicolumn{2}{|c|}{ Vehicle 1} & \multicolumn{2}{|c|}{ Vehicle 2} & \multicolumn{2}{|c|}{ Vehicle 3} & \multicolumn{2}{|c|}{ Vehicle 4} \\
\hline Observations & \multicolumn{2}{|c|}{492} & \multicolumn{2}{|c|}{592} & \multicolumn{2}{|c|}{421} & \multicolumn{2}{|c|}{450} \\
\hline Multiple $R$ & \multicolumn{2}{|c|}{0.847} & \multicolumn{2}{|c|}{0.721} & \multicolumn{2}{|c|}{0.869} & \multicolumn{2}{|c|}{0.786} \\
\hline$R$ square & \multicolumn{2}{|c|}{0.718} & \multicolumn{2}{|c|}{0.520} & \multicolumn{2}{|c|}{0.755} & \multicolumn{2}{|c|}{0.617} \\
\hline Adjusted $R$ square & \multicolumn{2}{|c|}{0.717} & \multicolumn{2}{|c|}{0.519} & \multicolumn{2}{|c|}{0.754} & \multicolumn{2}{|c|}{0.616} \\
\hline \multirow[t]{2}{*}{ F stat } & \multicolumn{2}{|c|}{622.926} & \multicolumn{2}{|c|}{319.211} & \multicolumn{2}{|c|}{644.126} & \multicolumn{2}{|c|}{360.712} \\
\hline & Coefficients & $t$ stat & Coefficients & $t$ stat & Coefficients & t stat & Coefficients & $t$ stat \\
\hline Intercept & 2.024 & 5.795 & -1.390 & -2.853 & 0.733 & 2.124 & 0.796 & 1.703 \\
\hline Magnitude & 3.190 & 27.398 & 6.138 & 20.042 & 2.984 & 29.934 & 3.823 & 19.854 \\
\hline Average speed & -0.038 & -7.370 & -0.030 & -6.673 & -0.013 & -2.558 & -0.021 & -3.503 \\
\hline
\end{tabular}

good, and there are no big differences among them (Figure 4).

However, under more realistic setting of the smartphones, which is the scope of this study, for the arrangement where the sum of magnitudes is calculated from the sum of all axes acceleration vibration, there are mixed results of the $R^{2}$ values in the total range of frequency ( 0 to $50 \mathrm{~Hz}$ ). As shown in Figures 5 and 6 , while the $R^{2}$ values are generally good for most vehicles using smartphone $\mathrm{C}$, except vehicle $1 \mathrm{~b}$ where the $R^{2}$ value is poor, the $R^{2}$ values for most vehicles that use smartphone D are poor, with also one exception that has a slightly bigger $R^{2}$ value (vehicle 3 ). The derived $R^{2}$ values in the case of smartphone D are relatively small which may be related to its location. In the box near the gearshift, the smartphone D is more likely to absorb the noise mainly from the vehicle engine (which is completely irrelevant to the vibration caused by road surface roughness), while smartphone $\mathrm{C}$ is more likely to absorb the noise mainly from the movement of the driver (which is still often and somehow related to the vibration caused by road surface roughness). Therefore, smartphone D may have absorbed more irrelevant noise than smartphone C. This may explain why $R^{2}$ in the case of smartphone $C$ is greater than that of smartphone $\mathrm{D}$.

Note that in Figures 5 and 6, Veh $1 \mathrm{p}$ is vehicle 1 with the smartphone in the pocket; Veh $1 \mathrm{~b}$ is also vehicle 1 but the smartphone is in the box near the gearshift; and Veh 2, Veh 3, and Veh 4 are vehicle 2, vehicle 3, and vehicle 4 , respectively.

By investigating further, taking into account the different ranges of frequency, it has been revealed that, for all cases, the effect of road roughness apparently occurs at high frequency ranges. The $R^{2}$ values in low frequency ranges are relatively smaller than the $R^{2}$ values in higher frequency ranges. Selected results are shown in Figure 7.

At the frequency range of 40 to $50 \mathrm{~Hz}$, the $R^{2}$ of the correlation between magnitudes and average IRI appears to be the strongest. Therefore, we believe that this frequency range is the most useful range that can be used to estimate road roughness condition (IRI) from acceleration data obtained by smartphone sensors, particularly when the smartphone is not fixed.

Comparing Figure 5 to Figure 8 and Figure 6 to Figure 9, respectively, it is very obvious that better $R^{2}$ results are derived in the frequency range of 40 to $50 \mathrm{~Hz}$, particularly for smartphone D. We assume that irrelevant noises, which is caused by the vehicle engine and the movement of the driver, for instance, may occur at low frequency ranges. Therefore, at the frequency range of 40 to $50 \mathrm{~Hz}$, those noises are not likely to have caused significant effect, leaving mainly the vibration signal that is generated by the road roughness condition.

A further investigation into the correlations between each axis of the acceleration vibration and IRI has also been done. Similar results, as discussed above, have been observed. There is no big difference in the correlation between the magnitudes and IRI in the total and breakdown frequency ranges, in the case of the smartphones that have been fixed; for the smartphones that have been placed at location with realistic settings, a frequency range of 40 to $50 \mathrm{~Hz}$ is the most useful range that can be used to estimate IRI.

A statistical analysis is also performed; it reveals that the speed of the vehicle also plays a role in the relationship between the acceleration data and the road roughness condition. Adjusted $R^{2}$ for all the cases are still significant and close to the original $R^{2}$ values. A selected summary of the multiple regression analysis is shown in Table 3.

\subsection{Estimation of IRI from the magnitudes calculated from the sum of all axes acceleration vibration}

A multiple linear model is considered, where the magnitudes calculated from the sum of all axes acceleration vibration and average speed are set as explanatory variables. The fit of the model is demonstrated in Figure 10. 


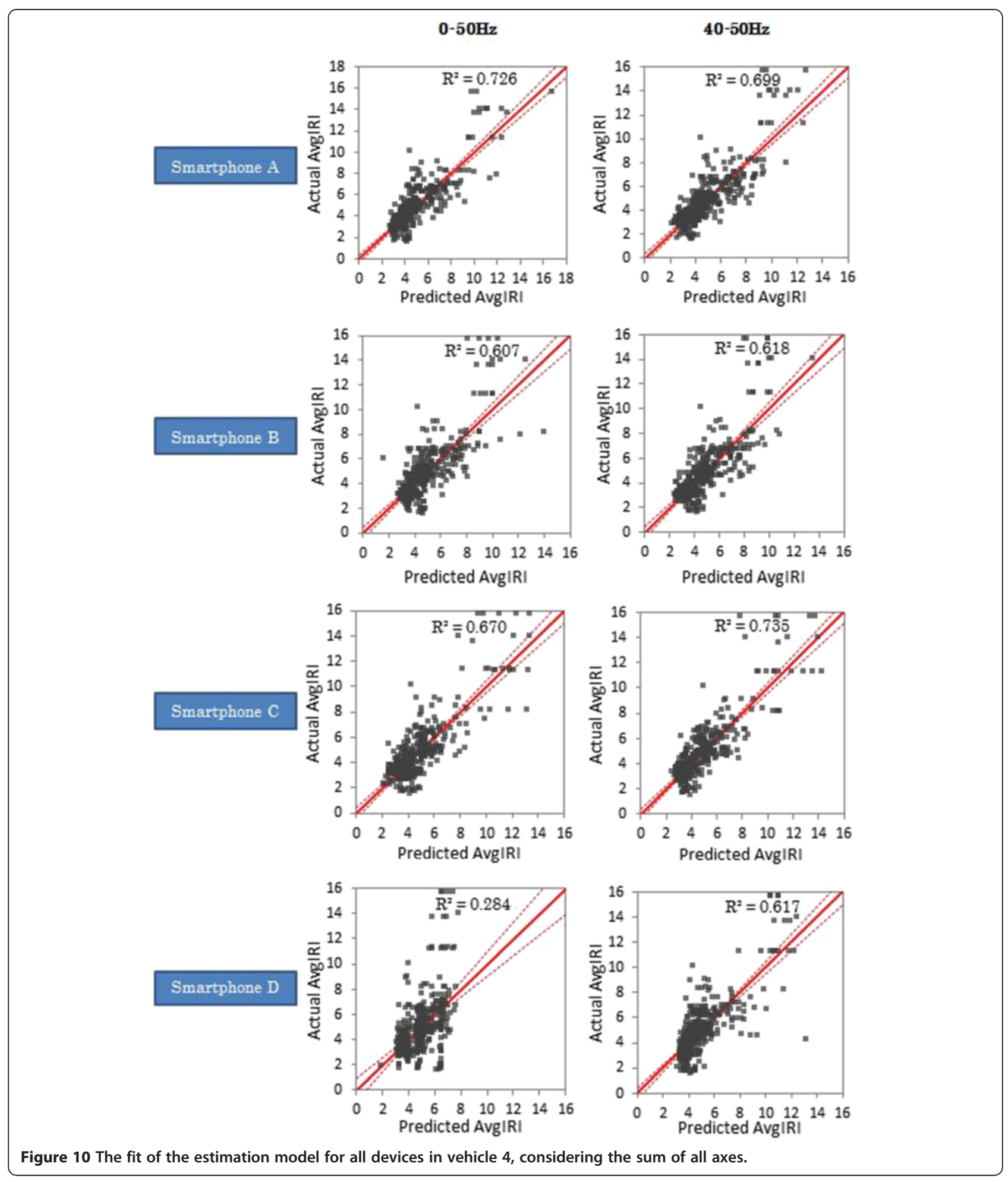

4.3 Estimation of IRI from the magnitudes calculated from each axis of acceleration vibration separately

Similarly, Figure 11 shows the fit of the estimation model when considering the sum of magnitudes calculated from each axis of acceleration vibration and average speed as explanatory variables in the model to predict IRI.

As Figures 10 and 11 indicate, better fit of the model can be achieved when considering each axis of acceleration vibration separately as variables, rather than the 


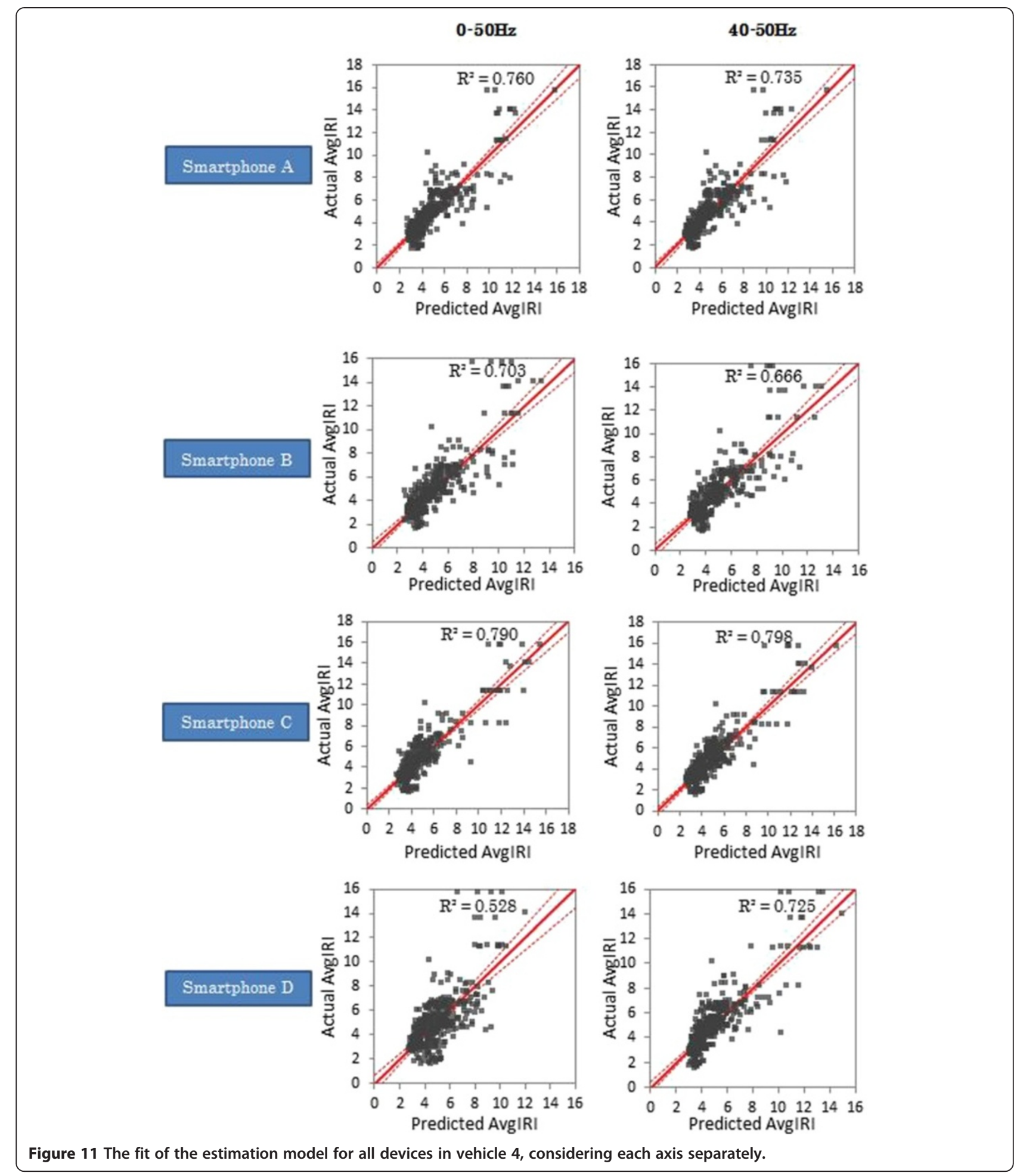

sum of all axes. Furthermore, when comparing Figure 11 to Figure 10, by device, respectively, Figure 11 shows better $R^{2}$ both in the total frequency ranges $(0$ to $50 \mathrm{~Hz})$ as well as in frequency range of 40 to $50 \mathrm{~Hz}$. These two figures also confirm that, for the smartphones that have been placed at locations under realistic settings, the frequency range of 40 to $50 \mathrm{~Hz}$ is more appropriate to be used for the estimation of IRI.

\section{Conclusions}

To further explore the use of smartphones to estimate road roughness condition from what we have left in our 
previous study, in this paper, we use smartphones to collect data for our analysis. The smartphones are placed loosely at locations such as inside the driver's shirt front pocket and the box near the vehicle gearshift. Similar to our previous study, after obtaining data from the experiment, data has been checked, filtered, matched with referenced data, and sectioned. The selected sections are then analyzed in frequency domain to calculate magnitudes of the signal in different frequency ranges. The relationship between the magnitudes and road roughness is investigated. The results of the study confirm that road roughness condition is linked to a linear function of magnitude of acceleration and average speed. It has also been revealed that, for the smartphones that have been placed at locations under realistic setting, in particular, vibration signal of the corresponding road pavement condition (roughness) occurs at the frequency range of 40 to $50 \mathrm{~Hz}$. In other words, data from the smartphone acceleration sensors at the frequency range of 40 to $50 \mathrm{~Hz}$ is best in expressing the road roughness condition. In regard to the model to be used for the estimation of IRI, it is more accurate to consider each axis of acceleration vibration as an explanatory variable separately.

In our key ongoing and future work, we are considering using many more different types of smartphones and vehicles as well as different realistic smartphone settings in our experiments. With these ongoing and future experiments, we believe that we will be able to understand more features and aspects on the use of smartphones for the estimation of road roughness condition. Additionally, we are also in the process of formulating a simple model and an Android application to estimate road roughness condition from many anonymous smartphones from real road users. After testing and fine tuning, we are planning to conduct a road condition estimation trial, where we would like to involve the participation from real road users, in Vientiane, Laos, in the very near future.

\section{Competing interests}

The authors declare that they have no competing interests.

Received: 31 January 2014 Accepted: 30 June 2014

Published: 11 July 2014

\section{References}

1. PD Hunt, JM Bunker, Analysis of unbound granular pavement deterioration for use in asset management modelling. (2001). http://eprints.qut.edu.au/ 7851/1/7851.pdf. Accessed 25 Aug 2013

2. MW Sayer, TD Gillespie, CAV Queiros, International road roughness experiment (The World Bank, 1986). http://deepblue.lib.umich.edu/bitstream/handle/ 2027.42/3134/72773.pdf;jsessionid=3C142BE33BAAF755253FF9DC82FEE683A? sequence $=2$. Accessed 19 Sept 2012

3. V Douangphachanh, H Oneyama, A study on the use of smartphones for road roughness condition estimation. J Eastern Asia Soc Trans Stud 10, 1551-1564 (2013)

4. A González, EJ O'brien, YY Li, K Cashell, The use of vehicle acceleration measurements to estimate road roughness. Veh Syst Dyn 46(6), 483-499 (2008)
5. J Eriksson, L Girod, B Hull, R Newton, S Madden, H Balakrishnan, The pothole patrol: using a mobile sensor network for road surface monitoring. Paper presented at the sixth international conference on mobile system, applications and services, Breckenridge, Colorado, United States, 2008.

6. P Mohan, VN Padmanabhan, R Ramjee, Nericell: Rich monitoring of road and traffic condition using mobile smartphones, in Proceedings of the $6^{\text {th }}$ ACM Conference on Embedded Network Sensor Systems, ed. by 2008, pp. 323-336

7. A Mednis, G Strazdins, R Zviedris, G Kanonirs, L Selavo, Real time pothole detection using Android smartphones with accelerometers. Paper presented at the 2011 international conference on distributed computing in sensor systems, Barcelona, Spain, 2011.

8. G Strazdins, A Mednis, G Kanonirs, R Zviedris, L Selavo, Towards vehicular sensor networks with Android smartphones for road surface monitoring. Paper presented at the 2nd international workshop on networks of cooperating objects, Chicago, USA, 2011.

9. Y Tai, C Chan, JY Hsu, Automatic road anomaly detection using smart mobile device. Paper presented at the 2010 conference on technologies and applications of artificial intelligence, Hsinchu, Taiwan, 2010.

10. M Perttunen, O Mazhelis, F Cong, M Kauppila, T Leppänen, J Kantola, J Collin, S Pirttikangas, J Haverinen, T Ristaniemi, Distributed road surface condition monitoring using mobile phones, Ubiquitous Intelligence and Computing, 2011, pp. 64-78

11. Android Application, BumpRecorder, Google Play Store. https://play.google. com/store/apps/details?id=jp.traffichazard.BumpRecorder. Accessed 8 Feb 2013

12. Android Application, AndroSensor, Google Play Store. https://play.google. com/store/apps/details?id=com.fivasim.androsensor\&hl=en. Accessed 12 Sept 2012

13. Vehicle Intelligent Monitoring System, VIMS, Bridge and Structure Laboratory (The University of Tokyo, Japan, 2012)

14. VIMS Manual, VIMS Consortium (Nagasaki University, Japan, 2012)

15. Android Developer: Android Developer Reference, SensorEvent, Android Developer, Android Developer Reference, SensorEvent. (2012). http:// developer.android.com/reference/android/hardware/SensorEvent.html. Accessed 26 Sept 2012

doi:10.1186/1687-1499-2014-114

Cite this article as: Douangphachanh and Oneyama: A study on the use of smartphones under realistic settings to estimate road roughness condition. EURASIP Journal on Wireless Communications and Networking 2014 2014:114

\section{Submit your manuscript to a SpringerOpen ${ }^{\circ}$ journal and benefit from:}

- Convenient online submission

- Rigorous peer review

- Immediate publication on acceptance

- Open access: articles freely available online

- High visibility within the field

- Retaining the copyright to your article

Submit your next manuscript at $>$ springeropen.com 